\title{
Programas Europeos de Intervención para Hombres que Ejercen Violencia de Género: Panorámica y Criterios de Calidad
}

\section{European Intervention Programmes for Men who Use Domestic Violence: Overview and Standards}

\author{
Heinrich Geldschläger \\ Fundación Instituto de Reinserción Social, Barcelona \\ Stefan Beckmann, Ludger Jungnitz y Ralf Puchert \\ Dissens e.V., Berlín, Alemania \\ Ansis Jurgis Stabingis \\ A.J. Stabinga konsultãcijas, Jurmala, Letonia \\ Cyril Dully \\ MOVE, Athlone, Irlanda \\ Heinrich Kraus \\ Centro de Hombres de Viena, Austria \\ Rosa Logar \\ Centro de Intervención en Abuso Doméstico, Viena, Austria \\ Per Kristian Dotterud y Jørgen Lorentzen \\ Reform-Centro de Recursos para Hombres, Oslo, Noruega \\ Sibylle Schweier
}

Federación Nacional de Asociaciones y Centros de Trabajo con Perpetradores de Violencia de Pareja y Familiar, Paris, Francia

\begin{abstract}
Resumen. En Europa, los primeros programas de intervención para hombres que ejercen violencia de género se iniciaron hace unos 25 años. Hasta ahora no se disponía de información sobre las diferentes características de estos programas en los diferentes países de la Unión Europea. Con el objetivo de ofrecer esta información y de promover el intercambio y el diálogo entre los programas europeos se realizó el proyecto Daphne "Trabajo con hombres que ejercen violencia doméstica en Europa". En este artículo se resumen dos de sus principales productos: los resultados de una encuesta en la que participaron 170 programas para maltratadores de 19 países y unas directrices para el desarrollo de estándares de calidad para este tipo de programas.

Palabras clave: violencia de género, intervención, hombres, maltratadores, Europa.
\end{abstract}

\begin{abstract}
The first intervention programmes for men who perpetrate domestic violence started in Europe about 25 years ago. Until now, little information has been available about the different characteristics of these programmes in different countries of the European Union. The Daphne project "Work with perpetrators of domestic violence in Europe - WWP" had the objective of providing this information and to promote exchange and dialogue between European programmes. This article summarizes two of the project's main outcomes: the results of a survey of 170 domestic violence intervention programmes from 19 European countries, and guidelines for the development of standards for these kinds of programmes.

Key words: domestic violence, batterer, intervention, programmes, Europe.
\end{abstract}

\section{Introducción}

Los programas de intervención con hombres que ejercen violencia contra sus parejas o ex -parejas son una de las posibles medidas para combatir la violen-

La correspondencia sobre este artículo puede dirigirse al primer autor a la Fundación Instituto de Reinserción Social (IReS). c/ Àlaba, 61, $1^{\circ} .08005$ Barcelona. E-mail: heinrich@iresweb.org cia de género y, potencialmente, mejorar la seguridad y el bienestar de las mujeres y de las hijas e hijos que la sufren. Organismos internacionales como la Plataforma de Acción de la cuarta Conferencia Mundial sobre Mujeres de la ONU en Beijing (1995) y el Comité de Ministros del Consejo de Europa en sus recomendaciones $n^{\circ} R$ (2002) 5 sobre la protección de las mujeres contra la violencia recomiendan a gobiernos, administraciones públicas y organizaciones 
comunitarias ofrecer y fomentar este tipo de programas y la investigación sobre ellos.

Los primeros programas de intervención con maltratadores (en inglés: "batterer intervention programmes") se crearon en EEUU en 1977 en respuesta al movimiento de mujeres contra la violencia de género y a los primeros servicios de apoyo para mujeres que habían sufrido violencia por sus parejas (Mederos, 2002). Durante los años 80 del siglo pasado estos programas se extendieron en el mundo anglo-sajón, en Latinoamérica y en Europa. En España hubo las primeras experiencias pioneras a finales de los $80 \mathrm{y}$ el primer programa empezó a funcionar a principios de los 90 .

\section{Objetivos}

A pesar de que existen, en Europa, programas para hombres que ejercen violencia de género con más de 20 años de trayectoria y experiencia, no se disponía de información centralizada sobre el estado de cuestión en cada país de la Unión Europea que permitiera sistematizar las diferentes experiencias y modelos e iniciar un proceso de intercambio y diálogo que facilitara una posible armonización de metodologías, contenidos y criterios de calidad de los programas. Crear esta información, iniciar el intercambio europeo y avanzar hacia unos criterios de calidad comunes fueron tres de los principales objetivos del proyecto europeo "Trabajo con Hombres que ejercen Violencia Doméstica en Europa" (Work with Perpetrators of Domestic Violence in Europe-WWP) cuyos resultados se resumen en este artículo. El proyecto fue realizado entre abril 2006 y abril 2008 por ocho entidades europeas en el marco del programa Daphne II para combatir la violencia contra niñas/os, jóvenes y mujeres y cofinanciado por el Ministerio Federal Alemán de la Familia, las Personas Mayores, las Mujeres y la Juventud.

\section{Metodología}

Se diseñó un cuestionario que abarcara la máxima información relevante sin ser demasiado largo y que se adaptara a los diferentes contextos para poder ser respondido por programas en los 27 países de la Unión Europea. El cuestionario contiene 50 preguntas divididas en ocho secciones: 1. Información Básica, 2. Personal, 3. Financiación, 4. Cooperación y Contexto (incl. Derivación y Acogida, Metodología), 5. Contenido del Trabajo, 6. Contacto con la Pareja y Apoyo y Seguridad para las Víctimas, 7. Calidad / Documentación / Evaluación y 8. Preguntas finales y Comentarios.
$\mathrm{Al}$ inicio del proyecto se decidió limitar la encuesta a aquellos programas de intervención con hombres que ejercen violencia de género que se realizaran fuera de los centros penitenciarios. Para identificar a los programas se utilizaron diferentes estrategias según el país, incluyendo redes formales e informales relevantes, documentación nacional e internacional sobre la temática, búsquedas a través de Internet, los colegios de psicólogos, institutos de la mujer, instituciones públicas relacionadas con la violencia de género (como, por ejemplo, observatorios, etc.) y otras.

A los programas identificados se les pidió que rellenaran el cuestionario online en la página Web de la encuesta facilitándoles una llave de acceso. El trabajo de campo, es decir la recogida de los datos a través de los cuestionarios, se llevó a cabo durante todo el año 2007. Un total de 170 programas de 19 países rellenaron el cuestionario.

Además, el mismo consorcio del proyecto "Trabajo con Hombres que ejercen Violencia Doméstica en Europa" desarrolló unas "Directrices para el desarrollo de estándares de calidad para los programas dirigidos a hombres que ejercen violencia doméstica" que se discutieron y validaron en una jornada de expertos realizada en Berlín en enero del 2008.

\section{Resultados}

A continuación se resumen dos de los principales productos del proyecto: primero un resumen por países de los principales resultados de la encuesta realizada y, después, las directrices para el desarrollo de estándares de calidad. Tanto un resumen más amplio de los resultados de la encuesta por países como las directrices para el desarrollo de estándares de calidad se encuentran disponibles en la página Web del proyecto (www.work-with-perpetrators.eu), conjuntamente con otros recursos.

\section{Resultados de la encuesta}

En los siguientes apartados se resumen muy brevemente y para cada país los principales resultados de la encuesta sobre los programas de intervención con hombres que ejercen violencia de género contra sus parejas. Para los siguientes cinco países no se consiguió identificar ningún programa en el momento de cerrar el trabajo de campo, a finales del 2007: Eslovaquia, Grecia, Italia, Letonia y Lituania. Además, en la República Checa la ONG "League of open men" ("Liga de los Hombres Abiertos") estaba planificando la puesta en marcha de un programa. 


\section{Alemania}

66 programas participaron. Los Programas para Perpetradores (a partir de ahora: PP) forman parte, en su mayoría, de una estrategia de intervención interinstitucional contra la Violencia Doméstica (a partir de ahora: VD) (p. ej, mesas redondas). La mayoría de los PP trabaja con hombres obligados por mandato judicial, y con hombres que acuden voluntariamente. La mayoría de los participantes lo hacen por obligación. Enfoque: La mayoría cognitivo conductual y/o enfoque sistémico (a veces mezclados). Financiación: Sólo $1 / 5$ de los PPs cuenta con una financiación permanente. La mayoría obtiene fondos en base a proyectos. A menudo, hay una combinación de financiación regional, municipal y privada. Dos de cada tres obtienen contribuciones por parte de los clientes. Apoyo a las víctimas: Más de $2 / 3$ de los PPs establecen contacto con la pareja del cliente, pero una cuarta parte de los mismos no ofrece ningún servicio de apoyo para la pareja del hombre que participa en el PP. De los PPs que contactan con la pareja, 2/5 ofrecen servicios de apoyo a la pareja a través de organizaciones colaboradoras; solo $1 / 7$ a través de la organización propia. Garantía de calidad y evaluación: 59 PPs tienen sesiones de equipo y 58 ofrecen supervisión. 36 PPs realizan evaluaciones internas, y 7 PPs han sido evaluados externamente.

Desde el año 2007, existe una organización central de los programas alemanes para perpetradores (www.taeterarbeit.com).

\section{Austria}

Ocho programas fueron identificados, seis cumplimentaron el cuestionario. Todos los PPs están ubicados en áreas urbanas o periurbanas y trabajan tanto con hombres que acuden voluntariamente como con los que lo hacen por requerimiento judicial. Enfoque: Los PPs austriacos trabajan con conceptos diferentes y en entornos variables. La mitad de los PPs no cuentan con ningún currículo en especial, ni con un sistema de documentación normalizado. Financiación: La mayoría de los fondos son para proyectos específicos, mezclados con financiación regional, municipal y gubernamental. Es obligatoria una contribución por parte del cliente. Apoyo a las víctimas: Únicamente uno o dos de los PPs ofrecen contacto con la pareja y sólo un programa, organizado por el Centro de Asesoramiento a los Hombres de Viena conjuntamente con el Centro de Maltrato Doméstico de Viena, ofrece un servicio de apoyo a las parejas. Solamente dos PPs realizan una colaboración y planificación conjunta y de manera regular con una organización de apoyo a las víctimas asociada. Garantía de calidad y evaluación: Ningún PP ha tenido una evaluación externa hasta el momento.

\section{Bélgica}

Tres programas participaron, dos en Valonia, uno en Flandes. Dos PP forman parte de una alianza interinstitucional contra la VD. Dos PP trabajan con hombres obligados por mandato judicial, y todos lo hacen con hombres que acuden voluntariamente o son derivados desde otras instituciones. En el 2006 trabajaron con menos de 25 , con 60 y con 984 hombres, respectivamente, todos en trabajo grupal, uno en trabajo individual y dos en asesoramiento conjunto con la pareja. Enfoque: Enfoque cognitivo conductual. Financiación: La financiación se distribuye por proyectos y es gubernamental, uno regional y uno se financia por la aportación de los clientes. Apoyo a las víctimas: Todos los PP contactan con la pareja del cliente para recabar información y colaboran con los servicios de apoyo a las víctimas. Garantía de calidad y evaluación: Todos cuentan con sesiones de equipo y supervisión. Dos miden los resultados de su trabajo, ninguno realiza evaluaciones de seguimiento, y únicamente dos hacen una evaluación externa.

\section{Chipre}

Se identificó un PP en Nikosia, parte de una alianza interinstitucional contra la violencia doméstica y que comenzó a trabajar en el 2007. Enfoque: Enfoque mixto con métodos sistémicos y cognitivo conductuales. Existen grupos cerrados que se reúnen semanalmente. Se usa un currículo e instrumentos de documentación estandarizados. Financiación: El PP se financia mediante fondos gubernamentales para proyectos específicos. No se obtienen contribuciones por parte de los clientes. Apoyo a las víctimas: El PP contacta con la pareja del cliente y ofrece servicios de apoyo desde la propia organización. También existe el intercambio de información. Garantía de calidad y evaluaciones: Debido a que el PP ha sido creado recientemente, ninguna evaluación externa ha sido realizada.

\section{Croacia}

En el 2003, se inició un proyecto piloto para perpetradores por parte de la ONG Society for Psychological Assistance (Sociedad para la Asistencia Psicológica). Dicha ONG ejecuta PPs en dos ciudades como parte de las medidas estatales contra la VD. Los participantes son derivados desde el sistema criminal judicial. Enfoque: Modelo cognitivo conductual con un currículo por escrito y grupos cerrados que se reúnen semanalmente durante dos horas. Financiación: En base a los proyectos; existe una cofinanciación por parte del gobierno y los municipios, pero es insuficiente. No se obtienen contribuciones por parte de los clientes. 
Apoyo a las víctimas: Los PPs contactan con la pareja del cliente. Servicios de apoyo son prestados por los socios de la misma organización.

\section{Dinamarca}

Tres programas participaron. Los PPs son prestados por parte de ONGs y trabajan tanto con hombres que acuden voluntariamente como con los que lo hacen por orden judicial. Enfoque: Psicodinámico (modelos ATV y DAVD) y la PNL (programación neurolingüística). Financiación: Todos los PPs reciben financiación privada, gubernamental o municipal. Apoyo a las víctimas: Dos PPs ofrecen servicios de apoyo a las víctimas, uno no lo hace.

\section{Escocia}

Entre 10 y 12 de los 32 Servicios de Justicia Penal de las autoridades locales cuentan con PPs. Uno de ellos es una colaboración con la ONG Sacro. En Edimburgo hay un programa que funciona al margen del sistema judicial, el Working with Men Project (Proyecto Trabajando con Hombres). El desarrollo de los PPs es parte clave del Plan escocés contra el Maltrato Doméstico con Menores y Jóvenes. Hay una red de servicios de apoyo para las mujeres (casas de acogida, etc.). Gran parte de los PPs se adhieren a las normas de Respect y ofrecen servicios integrados a las mujeres, aunque la financiación es problemática. CHANGE, DVPP y Sacro están desarrollando el Caledonian System (Sistema Caledoniano), un enfoque integrado dirigido al maltrato doméstico por parte de los hombres, para aumentar la protección de las mujeres y de los y las menores. Enfoque: Mayoritariamente, el enfoque es cognitivo conductual / de aprendizaje social dentro de un marco feminista. Financiación: Los PPs que son parte del sistema de justicia criminal (libertad condicional) reciben financiación del gobierno escocés. Los servicios para las mujeres y los y las menores reciben financiamiento del Fondo de la Violencia Contra las Mujeres. Working with Men recibe financiación de la Lotería Nacional y las autoridades locales. El gobierno escocés subvenciona el desarrollo del Sistema Caledoniano.

\section{Eslovenia}

Fue identificado un PP, ubicado en Ljubljana. El PP trabaja con ambos tipos de participantes: los obligados por mandato judicial y los que acuden por voluntad propia. Enfoque: Terapia cognitivo conductual sin un currículo por escrito. Financiación: El PP se basa en proyectos y recibe financiación gubernamental y municipal. No se obtienen contribuciones por parte de los clientes. Apoyo a las víctimas: El PP no contacta con la pareja del cliente de manera sistemática, pero hay un servicio de apoyo aparte, dentro de la misma organización, a disposición de las parejas/ex parejas de los clientes.

\section{España}

La totalidad de los 30 programas contactados respondieron. La mitad de los mismos comenzaron en el 2005 o posteriormente (después de la Ley de la Violencia de Género 1/2004). Tres de cada cuatro PPs son parte de una alianza entre agencias contra la VD. Más de las dos terceras partes de los PP trabajan con hombres que acuden por mandato judicial y por voluntad propia, pero la mayoría de los participantes lo hacen por obligación, y la mitad de los programas tienen un $80 \%$ de su clientela por mandato judicial. Enfoque: La mitad de los programas aplican el enfoque cognitivo conductual y la otra mitad otros enfoques (de género, sistémico, ecléctico, o integral, etc.). El 70\% ofrece trabajo grupal y uno de cuatro ofrece terapia de pareja (menos que en la totalidad del compendio de datos). Financiación: La mayor parte de la financiación es regional $(70 \%)$ o municipal $(20 \%)$, y únicamente $10 \%$ de los programas cobran honorarios. Sólo una tercera parte de los PPs cuentan con una financiación permanente, mientras que la mitad cuentan con financiación en base a proyectos. Apoyo a las víctimas: El 60\% de los PPs establece contacto con la pareja actual del cliente, y sólo $60 \%$ ofrecen servicios de apoyo a la pareja (40\% a través de la propia organización, y $20 \%$ a través de entidades colaboradoras). Garantía de calidad y evaluación: Sólo la mitad de los PPs cuentan con supervisión. Casi el 90\% miden los resultados de su trabajo, y el $60 \%$ realizan mediciones de seguimiento (el $40 \%$ las realiza dos veces), pero sólo $10 \%$ han sido objeto de una evaluación externa.

\section{Finlandia}

Un programa gestionado por el centro móvil contra las crisis (Universidad de Jyväskylä). El PP trabaja con hombres que acuden voluntariamente, tanto como con los derivados desde otras instituciones. Enfoque: Psicodinámico, modelo Alternatives to Violence (a partir de ahora: ATV). Financiación: Fondos regionales. No hay ninguna contribución por parte del cliente. Apoyo a las víctimas: Sí.

\section{Francia}

30 programas fueron identificados, 22 de los cuales cumplimentaron el cuestionario; desde entonces, se han localizado a nueve PPs más. En gran parte, los PPs 
forman parte de una alianza interinstitucional contra la VD. Los PPs trabajan con hombres que acuden por orden judicial y algunos también con hombres que acceden voluntariamente o que vienen derivados desde otras instituciones. La mayoría de los PP trabajó en el 2006 con menos de 50 hombres, y algunos con, incluso, menos de 25 hombres, mediante trabajo en grupo y asesoramiento individual. Enfoque: Psicodinámico, cognitivo conductual y sistémico (a veces mezclados). Financiación: La financiación se basa en proyectos, y con frecuencia, es mixta (nacional, regional, municipal y la participación económica por parte del cliente). Apoyo a las víctimas: Menos de una tercera parte de los PP establecen contacto con la pareja del cliente, y ofrecen servicios de apoyo a las víctimas (integrado o a través de organizaciones colaboradoras). No existen intervenciones coordinadas (apoyo a las víctimas y PP) en Francia. Garantía de calidad y evaluación: Casi todos ofrecen sesiones de equipo, la mitad cuenta con supervisión. La mitad de los PP miden los resultados de su trabajo; casi ninguno realiza medidas de seguimiento y únicamente dos cuentan con una evaluación externa.

La organización central de los programas franceses para con perpetradores fue fundada en el 2003 (www.fnacav.fr).

\section{Hungría}

No se localizó ningún PP. Funciona una línea telefónica de atención a los perpetradores. Los hombres maltratadores podrán acudir a terapeutas individuales, que podrán o no tener una comprensión clara de la violencia doméstica desde la perspectiva de género o de los roles de género en general.

\section{Inglaterra y Gales}

La estimación es que existen, al menos, 450 programas operativos en Inglaterra y Gales: 42 de las áreas de Juzgados y libertad provisional, 34 programas integrados sobre la violencia doméstica, ocho programas contra la violencia doméstica a nivel de los Condados, cinco programas de las autoridades locales y de servicios para las prisiones, p. ej., puede haber hasta 25 programas solamente en Manchester. Todos los programas tienen trabajo en fases previas y posteriores. Enfoque: Psicodinámico/ comportamiento cognitivo/ análisis de género. Financiación: El Ministerio del Interior del Reino Unido y las autoridades locales aportan la financiación. Apoyo a las víctimas: se presta a través de los y las trabajadoras en la seguridad de las mujeres y a través de socios. Garantía de calidad y evaluaciones: Sesiones de equipo, supervisión, pruebas psicométricas desde el principio hasta el final.

Respect es la asociación de miembros del Reino
Unido para los programas para perpetradores de violencia doméstica y los servicios asociados (www.respect.uk.net). Son 70 organizaciones o grupos implicados, repartidos por Irlanda, Irlanda del Norte, Inglaterra, Escocia y Gales.

\section{Irlanda del Norte}

La organización Men Overcoming Violence (Hombres Superando la Violencia) establecida en 1997, gestiona cuatro programas en Belfast, Derry, Antrim y Portadown. La participación en los programas es obligatoria mediante mandato judicial, y se ofrece contacto con la pareja a través de los servicios complementarios para las mujeres. Los programas tienen una duración de seis meses. Enfoque: Educativo, psicodinámico, cognitivo-conductual y el análisis desde la perspectiva de género. Financiación: Gubernamental, basado en los proyectos. Garantía de calidad y evaluaciones: Sesiones de equipo y supervisión.

\section{Luxemburgo}

Un programa que trabaja principalmente con hombres derivados desde otras instituciones, tanto como con hombres obligados por mandato judicial y algunos que acuden voluntariamente; hasta 50 en el 2006, únicamente con asesoramiento individual. No hay ninguna participación en alianzas interinstitucionales contra la VD. Enfoque: Psicodinámico. Financiación: Financiación pública nacional permanente, y la participación de los clientes. Apoyo a las víctimas: No hay contactos con otros socios ni colaboraciones con servicios de apoyo a las víctimas. Garantía de calidad y evaluación: Sesiones de equipo, supervisión. Hay medición de los resultados, pero sin seguimiento ni evaluaciones.

\section{Malta}

Un programa para perpetradores en Malta, que forma parte de la Agencia Nacional del Bienestar Social (National Social Welfare Agency “APPOGG”).

\section{Noruega}

15 programas participaron. Los servicios de los PPs son prestados por parte del servicio público de Asesoramiento para las Familias, hospitales universitarios, ONGs, y servicios en las prisiones y para la libertad condicional. La mayoría de los PP trabaja con una mezcla de hombres que acuden por mandato judicial y por voluntad propia. Enfoque: Psicodinámico (modelo 
ATV), cognitivo (modelo Brøset) y modelos basados en el asesoramiento. Financiación: Todos los PPs reciben financiación gubernamental o municipal. Apoyo a las víctimas: 10 PPs ofrecen servicios de apoyo a las víctimas, dos establecen contacto con la pareja del cliente, y tres no ofrecen apoyo alguno.

\section{Polonia}

Desde los años 2000/1 se gestiona en el centro regional para las adicciones de la ciudad de Olsztyn un programa educativo para hombres perpetradores según el modelo Duluth, que está en funcionamiento actualmente.

\section{Portugal}

Respondieron cuatro de los cinco programas contactados. Tres de los mismos están íntimamente ligados a o ubicados en Departamentos de Psicología de diferentes Universidades, mientras que el cuarto programa se gestiona por parte de una ONG para un consejo municipal. Tres de los PPs son parte de una alianza de agencias contra la VD (p. ej., PIs, mesas redondas). Los cuatro programas trabajan tanto con hombres que acuden por mandato judicial como con los que lo hacen voluntariamente, y son algo pequeños en tamaño (atendieron a menos de 25 hombres en el último año). Enfoque: Tres de los programas aplican un enfoque cognitivo conductual, y el otro uno ecléctico/integral. Sólo dos programas ofrecen trabajo grupal, y dos también ofrecen terapia de pareja. Financiación: Gran parte de la financiación es privada (dos programas) o procede de otras fuentes (los cuatro PP) y dos cobran a los participantes. Sólo un PP cuenta con una financiación permanente, mientras que la mitad de la misma se basa en casos. Apoyo a las víctimas: Únicamente uno de los cuatro PPs no establece contacto con la pareja actual ni ofrece servicios de apoyo a las parejas. Garantía de calidad y evaluación: Solamente la mitad de los PP cuenta con supervisión. Tres de los cuatro PP miden los resultados de su trabajo y realizan seguimiento (y dos hacen un segundo seguimiento), pero ninguno ha tenido una evaluación externa.

\section{República de Irlanda}

MOVE Ireland (Men Overcoming Violent Emotions-Hombres Superando las Emociones Violentas) ofrece programas para perpetradores en 10 ubicaciones en todo el país. Gran parte del trabajo es voluntario. De manera similar, el Programa Contra la Violencia Doméstica del sureste, y el Programa de Intervención Contra la Violencia Doméstica del noreste (NEVDPI) gestionan programas en sus zonas res- pectivas (únicamente es obligatoria la asistencia en el caso de NEVDPI). Valoración previa a la admisión al grupo. Los programas varían en su duración, desde las 26 semanas a programas continuados. Enfoque: cognitivo conductual, educativo, psicodinámico y análisis de género. Financiación: Financiación amparada por la ley a través del Departamento de Justicia y otros departamentos gubernamentales. Apoyo a las víctimas: El contacto con la pareja es una parte integral del servicio. Garantía de calidad y evaluaciones: Sesiones de equipo, formación, supervisión y evaluaciones continuas.

\section{Suecia}

20 programas. Los PPs son gestionados a través de los servicios sociales y las prisiones / servicios de libertad condicional. La mayoría de los PP trabaja con hombres cuya participación es por mandato judicial tanto como con hombres que acuden voluntariamente. Enfoque: Terapia cognitivo conductual (modelo IDAP) y ecléctica. Financiación: Todos los PPs reciben financiación gubernamental o municipal. Apoyo a las víctimas: Todos los PPs ofrecen apoyo a las víctimas, exceptuando uno que establece contacto con la pareja del cliente.

\section{Suiza}

Fueron identificados 26 PPs, 19 de los cuales cumplimentaron la encuesta, y hay dos PPs más que trabajan exclusivamente con mujeres perpetradoras. Seis de los 19 PPs ofrecen tratamiento a mujeres perpetradoras también. Las 3/4 partes forman parte de alianzas interinstitucionales (p. ej., PIs, mesas redondas, etc.). Cinco PPs ofrecen servicios de apoyo a las mujeres víctimas de la VD, tres PPs para las víctimas masculinas. La mayoría de los PPs trabajan con hombres cuya participación es por mandato judicial y con hombres que acuden voluntariamente, pero la mayoría de los hombres lo hace por voluntad / derivación propia. Apoyo a las víctimas: Sólo 1/5 parte de los PPs establecen contacto con la pareja del cliente. No obstante, 17 PPs incluyen un servicio de apoyo a las víctimas, ocho a través de la propia organización, nueve a través de entidades colaboradoras. Garantía de calidad y evaluación: Gran parte realiza sesiones de equipo, algo más que las $2 / 3$ cuenta con supervisión. Sólo $1 / 3$ ha tenido alguna evaluación interna, y ninguno de los PPs ha sido evaluado externamente.

\section{Directrices para el desarrollo de estándares de calidad para los programas dirigidos a hombres que ejercen violencia doméstica}

Las agencias que ejecutan programas dirigidos a los 
agresores asumen una gran responsabilidad ante todas las personas implicadas. El trabajo con los hombres que ejercen violencia doméstica tiene como objetivo poner fin a la violencia y aumentar la seguridad de las víctimas de la violencia doméstica (mujeres y niños/as), pero también debe interpretarse como integrado en un proceso más amplio de cambios culturales y políticos hacia la abolición de las jerarquías entre géneros, la violencia de género, y la discriminación de género, tanto como las demás formas de violencia y discriminación personal y estructural. Los estándares de calidad son necesarios para asegurar la calidad del trabajo y, particular y prioritariamente, la seguridad de las víctimas, y para que el trabajo realizado no suponga un peligro para las parejas o los/as hijos/as de los participantes. Las directrices que siguen para el desarrollo de unos estándares de calidad en el trabajo con los hombres agresores han sido recopilados por el consorcio del proyecto Daphne II "Work with Perpetrators of Domestic Violence in Europe-WWP" ("Trabajo con los Hombres que ejercen Violencia Doméstica en Europa"), y desarrollados durante una jornada de expertos internacionales celebrado en Berlín en enero del 2008. Las directrices se dirigen a programas con hombres que usan la violencia contra sus parejas y contra los y las menores que conviven con ellos.

Los programas existentes en los países europeos varían en cuanto a sus tareas, grupos destinatarios, financiación, base legal, y en muchos otros aspectos y condiciones de trabajo. Por tanto, estas directrices no pretenden ser unas instrucciones detalladas, sino, más bien, ofrecer a los programas con hombres agresores un marco de referencia para el desarrollo de unos estándares específicos para el ejercicio de un trabajo responsable. El apartado siguiente describe el objetivo y las precondiciones que deberán tenerse en cuenta para la realización de un trabajo responsable con hombres que ejercen violencia doméstica. Los estándares de calidad para el trabajo con los agresores deberán integrar las conclusiones nuevas de investigaciones y de las experiencias de buenas prácticas.

\section{A) Las condiciones previas para el trabajo con hombres que ejercen violencia doméstica}

\section{A.1) Objetivo}

El objetivo principal del trabajo con los hombres agresores es incrementar la seguridad de las víctimas de la violencia. Los programas dirigidos a los perpetradores deberán priorizar la seguridad de las parejas y de sus hijos/as a todos los niveles del programa. Dicho objetivo deberá ser explícito, tanto para los y las profesionales como para los hombres con los que trabajan.

\section{A.2) Colaboración con los servicios de apoyo a las víctimas y los sistemas de intervención}

Los programas dirigidos a los agresores son sólo una parte de un sistema necesariamente más amplio de intervención contra la violencia doméstica, y no deberán ejecutarse de manera aislada ni ser implementados donde sean inexistentes servicios de apoyo específicos para las víctimas. Los programas dirigidos a los agresores deberán financiarse con recursos adicionales y complementarios y no a costa de los servicios de apoyo a las víctimas.

Para tratar de manera efectiva la violencia doméstica, los programas dirigidos a los agresores deberán ser parte íntegra de un sistema de intervención y participar activamente en alianzas y redes de agencias contra la violencia doméstica. Es de importancia particular la cooperación estrecha con los servicios ofrecidos a las mujeres víctimas y a sus hijos/as, tanto para asegurar su seguridad como para lograr un tratamiento integral de la violencia doméstica. Estos principios de cooperación deberán implementarse mediante la inclusión de los representantes de los servicios de apoyo a las mujeres como expertos en comités de supervisión y en las juntas directivas de los programas dirigidos a los agresores. También son fundamentales la colaboración y la formación de redes con todos los demás servicios, agencias y profesionales que trabajan en el campo de la violencia doméstica (p. ej., el sistema judicial, los servicios sociales, los servicios sanitarios, y los servicios de protección de menores). La cooperación y la participación en las alianzas y redes deberán reconocerse y ser financiadas.

\section{A.3) Modelo teórico y concepto de trabajo explícito}

Los programas dirigidos a los agresores deben asentarse sobre un entendimiento y la actitud que la violencia contra las mujeres y los/as niños/as es inaceptable y que los hombres son responsables de su uso de la violencia. Todos los miembros del equipo profesional que trabajan con los perpetradores se abstendrán de justificar, tolerar o minimizar la violencia y condenarán cualquier forma de culpabilizar a la víctima. Además, cada programa que trabaja con los agresores necesita de una comprensión teórica explícita, que incluya los aspectos que siguen, sin ser excluyentes:

- teoría de género-comprensión de la jerarquía de género y de las masculinidades, incluyendo las influencias sociales, culturales, religiosas, étnicas y políticas,

- definición de la violencia doméstica y de los tipos de maltrato,

- orígenes de la violencia - comprensión de las causas y de los mecanismos que desembocan en la violencia,

- teoría de la intervención / teoría del cambio - 
comprensión de por qué se supone que las intervenciones propuestas cambian las actitudes y los comportamientos violentos de los participantes.

Dichas consideraciones teóricas deberán conducir a un concepto, explícito y presentado por escrito, sobre el trabajo con los agresores. Como guía para el desarrollo de tal concepto, están disponibles algunos modelos descritos detalladamente.

\section{A.4) Enfoque sobre las dimensiones relevantes para el uso de la violencia}

Para poder abordar de manera adecuada la complejidad de la violencia que los hombres utilizan contra sus parejas, la intervención deberá enfocarse sobre las dimensiones relevantes e importantes relacionadas con el fenómeno, que se pueden organizar en un modelo ecológico (Bronfenbrenner 1979, Dahlberg \& Krug 2002):

a) Factores socioculturales incluyendo el contexto social desde una perspectiva de género, las relaciones de poder desiguales entre hombres y mujeres en nuestras sociedades, la persistencia de la violencia como método para afrontar el conflicto en nuestras culturas, las sanciones sociales y del sistema judicial ante el uso de la violencia doméstica, etc.

b) Factores relacionales incluyendo las relaciones de poder en la pareja desde el enfoque de género, los patrones ante la solución de conflictos y de la comunicación, etc.

c) Factores personales del individuo que podrán dividirse en las categorías siguientes:

- Factores cognitivos incluyendo las creencias y las actitudes respecto de las relaciones y los roles de género, las expectativas de la relación (p. ej., el amor romántico), de la pareja y de sus hijos/as (p. ej., derecho a recibir servicios), y de uno mismo (p. ej., masculinidad e identidad).

- Factores emocionales incluyendo la regulación (identificación, comprensión y expresión) de los sentimientos de enfado, frustración, fracaso, vergüenza, envidia, miedo, etc., y su relación con el género y los patrones experienciales sobre los cuales se fundamentan (estilos de apego, sentido de la identidad, las expectativas, etc.).

- Factores conductuales incluyendo la sustitución de los comportamientos violentos y controladores basados en el género por habilidades y destrezas para una relación respetuosa e igualitaria, como son la empatía, la comunicación y la resolución de conflictos, la gestión del estrés y del enfado, etc.

\section{B) Principios importantes para el trabajo con los hombres agresores}

\section{B.1) Contacto con y apoyo para la pareja}

Para aumentar la seguridad de la pareja del agresor, los programas dirigidos a los agresores deberán asegurar que las parejas de los hombres son informadas de los objetivos y del contenido del programa, sobre sus limitaciones (p. ej., que no suponen una garantía de la no violencia), sobre cómo el hombre puede utilizar su participación en el programa para manipular o controlarla aún más, y respecto de sus propias posibilidades para recibir apoyo y proteger su seguridad. La información aportada por la pareja deberá incluirse en la valoración del riesgo y en la evaluación del agresor. Se deberá advertir a la mujer si su pareja deja de asistir al programa o si los facilitadores perciben que exista algún riesgo para la mujer o para sus hijos/as.

Deberá asegurarse que el contacto con la pareja sea completamente voluntaria para ellas, y que no implique ninguna responsabilidad en cuanto a la participación del agresor en el programa, o de su progreso en el mismo. Las necesidades de la mujer deberán ser respetadas, y se harán todos los esfuerzos para minimizar cualquier riesgo posible relacionado con el contacto con la pareja. El contacto con la pareja podrá facilitarse mediante un servicio de apoyo a las víctimas o por el mismo programa de atención a los hombres.

\section{B.2) Política de protección de menores}

Los/as niños/as que viven en relaciones de maltrato son siempre (directa o indirectamente) afectados por la violencia doméstica. Por tanto, los programas que trabajan con los agresores deberán priorizar la perspectiva de estos y estas menores, tanto en su actividad directa con los hombres como a nivel de su integración dentro de un sistema de intervención más amplio y en la cooperación con otras entidades. El programa deberá establecer una política específica sobre la protección de menores, que contemple pasos concretos a ejecutarse en el caso que un menor esté en riesgo, adaptada al contexto local y a la normativa legal. Los efectos de la violencia doméstica sobre los/as niños/as y el rol como padre de los participantes deberá formar parte del currículo de los programas dirigidos a los agresores.

\section{B.3) Enfoques y actitudes en el trabajo directo con los agresores}

Los programas dirigidos a los agresores se fundamentan en la creencia que las personas tienen la capacidad de cambiar. 
Los programas dirigidos a los agresores deberán responsabilizar a los hombres con los que trabajan por la violencia que utilizan y destacar la necesidad de que ellos asuman la responsabilidad por sus comportamientos violentos y las consecuencias de éstos. Aún así, es imprescindible que el o la profesional trate al agresor con respeto y como persona con valor intrínseco.

Uno de los supuestos básicos sobre los cuales fundamentar el programa deberá ser el uso de la violencia como una elección. Por tanto, uno de los primeros objetivos a lograr en el trabajo con los hombres deberá ser ayudarles a reconocer que eligen utilizar la violencia. Los discursos de negación, justificación, excusa o de culpabilizar a los demás o a las circunstancias deberán ser cuestionados y desconstruidos.

Un enfoque detallado sobre el comportamiento violento y la reconstrucción de los actos específicos, los pensamientos y los sentimientos, ayudarán a los hombres a reconocer su papel activo en el uso de la violencia. Una exploración pormenorizada de los muchos y diferentes impactos y las consecuencias que tiene la violencia sobre sus parejas e sus hijos/as ayudará a promover en los hombres la empatía, la responsabilidad y la motivación para cambiar.

Los y las profesionales deberán asegurar que los clientes, tanto las víctimas como los agresores, no sean dañados de ninguna manera por el trabajo y el enfoque del programa. Deberán reconocer que, en algunos casos (p. ej., cuando hay abuso del alcohol, trastornos de la personalidad) podrá ser más apropiado derivar a la persona a otro servicio más adecuado a sus necesidades individuales.

\section{B.4) Valoración del riesgo}

La valoración sistemática del riesgo deberá ser implementado. La identificación de los hombres con un riesgo elevado de ser violentos permite a los y las profesionales iniciar las medidas más adecuadas para asegurar la seguridad de las víctimas y aporta información importante respecto de las necesidades de tratamiento específicas. La valoración del riesgo deberá realizarse y ser documentada tanto durante la fase de admisión al programa como en cualquier momento que el comportamiento del agresor o la situación indique un posible cambio en cuanto al riesgo. En la valoración del riesgo deberá incluirse el mayor número de fuentes de información posibles, particularmente la perspectiva de la pareja, pero también informes policiales e información de otras agencias que atiendan al participante o a su familia. Se deberán tener en cuenta las limitaciones de la exactitud en las valoraciones del riesgo.

\section{B.5. Capacidad del equipo profesional}

Para tener un nivel de calidad elevado en el trabajo con los agresores, más allá de un entrenamiento básico, los miembros del equipo de profesionales deberán contar con las capacidades siguientes:

- Compromiso con las relaciones sin violencia y con la igualdad de género.

- Formación especializada en la sensibilización sobre la violencia.

- Auto reflexión sobre la relación personal en cuanto a los roles y las identidades de género y la sensibilidad ante las jerarquías de género y el sexismo.

- Auto análisis sobre la tendencia personal hacia el comportamiento violento o dominante, y concienciación sobre la historia personal de violencia.

- Comprensión exhaustiva de las dinámicas en las relaciones violentas.

- Formación y supervisión continua.

- Habilidades de conducción de grupos (para los facilitadores de grupos).

Si los o las facilitadores no son profesionales sujetos a un código ético regulador (p. ej., colegio de psicólogos), los temas éticos deberán regularse de manera específica, como son la confidencialidad, la protección de datos, la relación entre facilitador/a y participante, etc.

\section{B.6) Control de la calidad, documentación y evaluación}

El control de la calidad, la documentación y la evaluación del trabajo deberán ser parte integral de todos los programas. Los programas dirigidos a los agresores deberán documentar y evaluar sus procesos y resultados.

Los y las profesionales deberán crear e implementar unas medidas para la evaluación continua de los procesos y los resultados de su trabajo, y relacionarlos con las conclusiones de investigaciones y las buenas prácticas a nivel nacional, $y$, cuando sea posible, a nivel internacional. Estas medidas deberán incluir:

- supervisión y reuniones de equipo periódicos,

- la documentación del trabajo de forma continua,

- el análisis de dicha documentación,

- la evaluación interna y externa de los resultados del programa.

\section{C) Más información}

Estas directrices para los programas que trabajan con los hombres perpetradores de la violencia doméstica podrán utilizarse para el desarrollo o la revisión de los estándares de los programas. Enlaces a documentos existentes sobre estándares de calidad que pueden servir como ejemplo para una elaboración detallada de las mismas, y más información, pueden 
encontrarse en la página Web: www.work-with-perpe trators.eu

\section{Referencias}

Bronfenbrenner, U. (1979). The Ecology of Human Development: Experiments by Nature and Design. Cambridge, MA: Harvard University Press.

Council of Europe. (2004). The protection of women against violence: Recommendation No. R (2002) 5 adopted by the Committee of Ministers on 30 April 2002 and explanatory memorandum. Strasbourg: Council of Europe, Directorate General of Human Rights.
Dahlberg, L. L. y Krug, E. G. (2002). Violence: A Global Public Health Problem. En E. G. Krug, L. L. Dahlberg, J. A. Mercy, A. B. Zwi, \& R. Lozano (eds.): World Report on Violence and Health, (pp. 1-21). Geneva: World Health Organization.

Mederos, F. (2002). Changing our visions of interventionthe evolution of programs for physically abusive men. En E. Aldarondo, \& F. Mederos (Eds.), Men who batter: Intervention and prevention strategies in a diverse society ( pp. 1-1-1-26). New York: Civic Research Institute.

Organización de Naciones Unidas (1995). Declaración y Plataforma de Acción del cuarto Congreso Mundial sobre Mujeres en Beijing. Beijing: Naciones Unidas.

Manuscrito Recibido: 22/03/2010

Revisión Recibida: 08/05/2010

Manuscrito Aceptado: 08/05/2010 